\title{
Echo Delay and Overlap with Emitted Orientation Sounds and Doppler-shift Compensation in the Bat, Rhinolophus ferrumequinum *
}

\author{
Gerd Schuller \\ Arbeitsgruppe Neuro- und Rezeptorphysiologie, Fachbereich Biologie, Universität Frankfurt, \\ D-6000 Frankfurt, Federal Republic of Germany
}

Received September 17, 1976

Summary. The compensation of Doppler-shifts by the bat, Rhinolophus ferrumequinum, functions only when certain temporal relations between the echo and the emitted orientation sound are given. Three echo configurations were used:

a) Original orientation sounds were electronically Doppler-shifted and played back either cut at the beginning (variable delay) or at the end (variable duration) of the echo.

b) Artificial constant frequency echoes with variable delay or duration were clamped to the frequency of the emitted orientation sound at different Doppler-shifts.

c) The echoes were only partially Doppler-shifted and the Doppler-shifted component began after variable delays or had variable durations.

With increasing delay or decreasing duration of the Doppler-shifted echo the compensation amplitude for a sinusoidally modulated $+3 \mathrm{kHz}$ Dopplershift (modulation rate $0.08 \mathrm{~Hz}$ ) decreases for all stimulus configurations (Figs. 1, 2, 3).

The range of the Doppler-shift compensation system is therefore limited by the delay due to acoustic travel time to about $4 \mathrm{~m}$ distance between bat and target. In this range the overlap duration of the echo with the emitted orientation sound is always sufficiently long, when compared with data on the orientation pulse length during target approach from Schnitzler (1968) (Fig. 5).

\section{Introduction}

In free flight horseshoe bats, Rhinolophus ferrumequinum, emit sounds with durations up to $60 \mathrm{~ms}$ and therefore hear echoes which overlap with the emitted sounds when the target distance is less than $10 \mathrm{~m}$. The echolocation signal

\footnotetext{
* Supported by Stiftung Volkswagenwerk, grant No. 111858; Deutsche Forschungsgemeinschaft, grant No. Ne146/7, Schu 390/1, Schn 138/6
} 
consists of a long constant frequency (CF) component with an initial and final short frequency modulated (FM) component (Schnitzler, 1968). The possible significance for some echolocation task of the temporal overlap of the echoes with the emitted sounds was first proposed by Pye (1960). The importance of temporal overlap for the proper operation of the Doppler-shift compensation system in this bat was demonstrated by Schuller (1974). No overlap between echo and outgoing signal made Doppler-shift compensation impossible. When the echoes were delayed by more than $25 \mathrm{~ms}$ relative to the outgoing sound the compensation of the Doppler-shifts deteriorated dramatically. In these experiments the frequency of the Doppler-shifted artificial echoes did not change with compensation but always contained a $1 \mathrm{kHz}$ frequency-shift. The measurements yielded therefore only few quantitative data as the bats had only the possibility of detecting the Doppler-shift, but not of compensating for it. How the compensation deteriorates with increasing delay of the echoes or decreasing overlap time between echo and orientation sound is the subject of this paper.

\section{Methods}

The influence of temporal shifts and of varying overlap times between echoes and emitted orientation sounds on the Doppler-shift compensation was measured in three horseshoe bats, Rhinolophus ferrumequinum. All three bats were compensating normally with a $3 \mathrm{~ms}$ long delay between echo and emitted orientation sound produced by the acoustical delay between the bat and the microphone and loudspeaker assembly. The experimental arrangement and the method of simulating Dopplershifted echoes were identical to those described in previous experiments (Schuller et al., 1974). The temporal relationship between the Doppler-shifted echo and the emitted orientation sound was manipulated in three different ways:

a) Playback of Segmented Simulated Doppler-shifted Echoes. An electronic switch was used to play back only parts of the simulated echoes, so that the echo could be cut at the beginning and the end of the sound. The echo could therefore start after a controllable delay and could have a variable duration, but could never outlast the emitted orientation sound by more than $3 \mathrm{~ms}$.

b) Parially Doppler-shifted Echoes. In this situation the simulated echo started after a 3 ms delay and lasted as long as the emitted signal. Doppler-shifts could be introduced after variable delays and with different durations, so that the frequency in the simulated echo was partially equal to the emitted frequency and partially Doppler-shifted.

c) Simulated Echo without Frequency Modulated Components. An artificial constant frequency signal was used as a simulated echo. The time delay to the beginning of the emitted signal and the duration of the echo were varied, so that the artificial echo could also outlast the emitted signal. A voltage representing the $C F$ of the emitted sound was sampled $7.5 \mathrm{~ms}$ after the onset of the emitted signal and stored in a sample and hold circuit until the next sound was emitted. The frequency of the artificial echo was controlled by this voltage representing the $\mathrm{CF}$ of the emitted signal plus a voltage representing an upward Doppler-shift fed to the VCG-input of the sine wave generator (Wavetek 136). Thus the frequency of the artificial echo was clamped to the CF of the emitted signal and the Doppler-shift compensation system of the bat could function normally.

The CF of the emitted orientation sounds was recorded on a fast strip chart recorder (Hellige, Helcoscriptor HE-1). The influence of delay time and echo duration on the Doppler-shift compensation was measured for steady and sinusoidally modulated Doppler-shifts. 


\section{Results}

Playback of Segmented Simulated Doppler-shifted Echoes. As the orientation sounds of the horseshoe bat consist of frequency modulated components and a long constant frequency component the question arose whether the FM components play any role in the compensation of Doppler-shifts. Therefore the FM components in the simulated echoes were cut out by an electronic switch and only shortened constant frequency components were played back so that the $\mathrm{CF}$ of the echo did not overlap with the FM component of the orientation sound. No change in the Doppler-shift compensation could be detected under these conditions. The Doppler-shift compensation works without the FM components and only the CF component is necessary for the compensation system. This was confirmed by the playback of totally artificial CF tone bursts: the bats emitted the appropriate frequency to compensate for the apparent Dopplershift of their own echoes. As the artificial echoes were constant in sound pressure level (SPL) and did not follow the SPL of the emitted sounds the frequency parameter of the CF component is the important factor for compensation of Doppler-shifts.

When the frequency of the CF component of the simulated echoes is sinusoidally Doppler-shifted with a low modulation frequency $(0.08 \mathrm{~Hz})$ the bat responds by modulating its emitted frequency roughly sinusoidally in order to compensate for the shifts. The peak compensation, that is the difference between the emitted frequency at zero shift and that at the maximum positive Dopplershift, was used as a measure of the compensation performance. The sinusoidal modulation introduced Doppler-shifts between zero and $3 \mathrm{kHz}$ at a constant modulation period of $0.08 \mathrm{~Hz}$. At this modulation period the compensation amplitude is not limited by the speed of the control system for Doppler-shift compensation (Schuller et al., 1975).

For almost complete overlap ( $3 \mathrm{~ms}$ delay) between echoes and emitted orientation sounds the peak compensation was maximum and taken as the normal value. When the echoes were cut at the beginning, the peak compensation decreased with increasing amount of cut. At a delay of 20 to $30 \mathrm{~ms}$ modulation of the emitted frequency ceased. The compensation amplitude is represented in Figure $1 \mathrm{~A}$ for two bats as a function of the delay of the beginning of the echoes. The $-6 \mathrm{~dB}$ points lie at about 17.5 and $22.5 \mathrm{~ms}$.

When the echoes were shortened at the end, the peak compensation decreased with decreasing echolength and correspondingly smaller overlap. Figure 1B shows the compensation amplitude as a function of the echo length with $-6 \mathrm{~dB}$ points at 22 and $10 \mathrm{~ms}$. The compensation system in one bat (gr) works up to higher delays of the echo and with shorter overlaps between echo and emitted orientation sound than in the other bat $(\mathrm{gg})$. The characteristics of the decay of the compensation amplitude are substantially the same. Increasing delay causes a shortening of the echo duration if the duration of the orientation sound is constant. The duration of the orientation sounds was on average $50 \mathrm{~ms}$, so that the corresponding echo durations at the $-6 \mathrm{~dB}$ points for the delay measurements were 32.5 and $27.5 \mathrm{~ms}$, that is larger than the echo duration at the $-6 \mathrm{~dB}$ points of the echo duration measurements with a $3 \mathrm{~ms}$ delay 

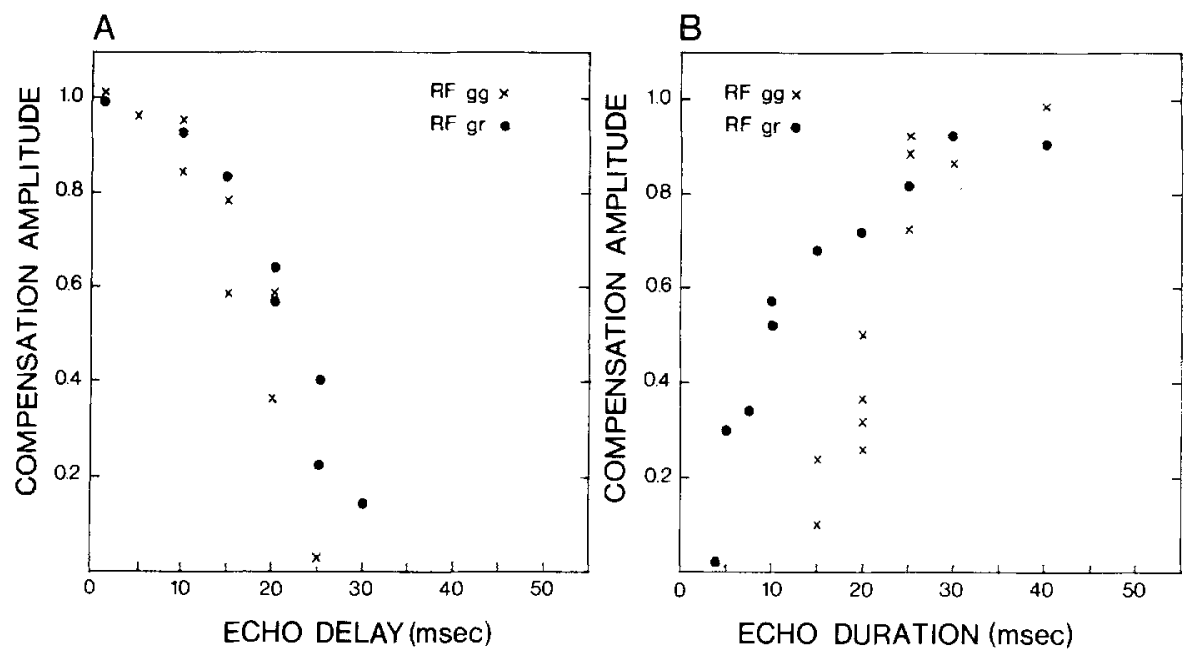

Fig. $1 \mathrm{~A}$ and B. Playback of segmented simulated Doppler-shifted echoes: Peak compensation amplitude for sinusoidal modulation between zero and a positive Doppler-shift (modulation rate $0.08 \mathrm{~Hz}$, max. pos. Doppler-shift $3 \mathrm{kHz}$ ) for two bats as a function of the echo delay to the beginning of the emitted orientation sound $\mathbf{A}$ and as a function of the duration of the echo starting $3 \mathrm{~ms}$ after the onset of the emitted orientation sound $\mathbf{B}$

of the echoes ( 22 and $10 \mathrm{~ms}$ ). The decrease of compensation amplitude with increasing delay time is not due to the decrease of corresponding echo duration, as the bats still reach full compensation for echo durations of 32.5 and $27.5 \mathrm{~ms}$ when the delay time is small (3 ms) (Fig. 1 B).

The SPL of the echoes was kept constant at $-25 \mathrm{~dB}$ relative to the emitted orientation sound during the measurement. The absolute SPL of the orientation sounds ranged from about 90 to $100 \mathrm{~dB}$ SPL $34 \mathrm{~cm}$ in front of the bat.

Playback of Artificial Sounds with Delay and Limited Overlap Time. This method differs from that described above by the lack of the frequency modulated portions in the echoes, the constant sound pressure level during the echoes and the fact that the artificial echoes can last longer than the emitted orientation sound. The measurement itself was similar to that used with segmental playback and was carried out with sinusoidal modulations of $0.08 \mathrm{~Hz}$ between zero and a positive $3 \mathrm{kHz}$ Doppler-shift. The results are given as plots of the normalized compensation amplitudes versus the delay of the echo relative to the emitted orientation sound or versus the duration of the echo from $3 \mathrm{~ms}$ after the onset of the emitted signal. Figure 2 gives an example how the compensation amplitude decreases with increasing delay between echo and echolocation signal. The normalized compensation amplitudes for three bats are combined in the plot of Figure $3 \mathrm{~A}$ and show a very similar decay with increasing delay. The $-6 \mathrm{~dB}$ values range between $12.5 \mathrm{~ms}$ and $17.5 \mathrm{~ms}$.

The normalized compensation amplitudes for echoes starting $3 \mathrm{~ms}$ after the onset of the orientation sound and with variable echo duration are represented as a function of the echo duration in Figure $3 \mathrm{~B}$. Here also the variations between individual bats are larger than in the delay measurements. 

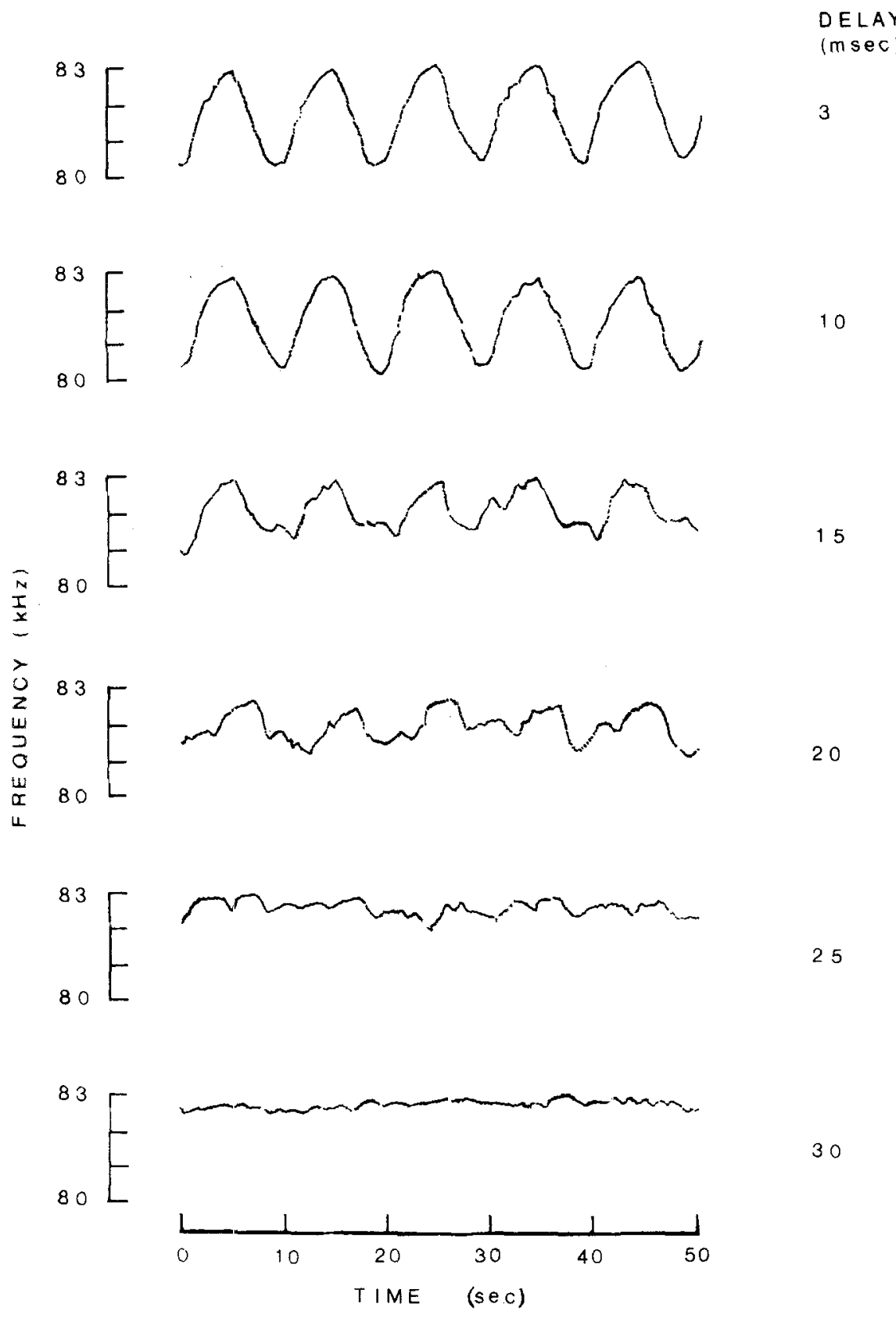

Fig. 2. Frequency of the CF component of the orientation sounds when the bat compensates for a sinusoidally modulated (rate $0.08 \mathrm{~Hz}$ ) frequency shift between zero and $+3 \mathrm{kHz}$. The parameter on the right side indicates the delay of the artificial frequency-shifted echo to the onset of the emitted orientation sound 

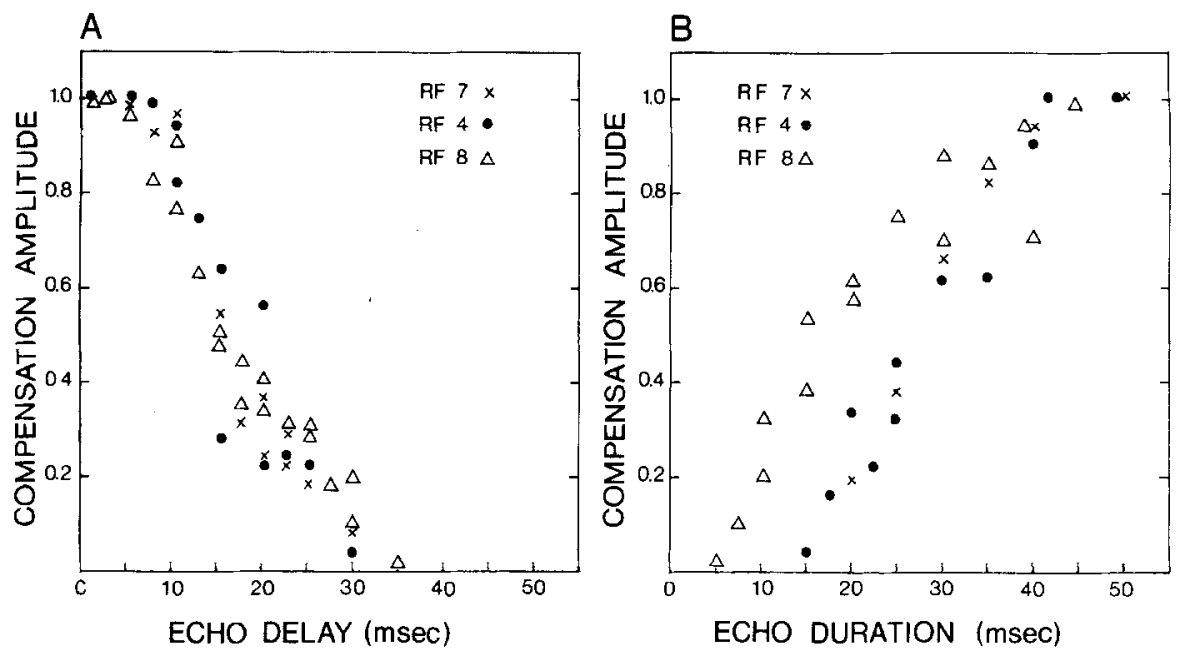

Fig. $3 \mathrm{~A}$ and B. Playback of artificial frequency shifted-echoes without frequency modulated components: Peak compensation amplitude for sinusoidal modulation between zero and a $+3 \mathrm{kHz}$ Doppler-shift (modulation rate $0.08 \mathrm{~Hz}$ ) for three bats as a function of the echo delay to the beginning of the emitted orientation sound $\mathbf{A}$ and as a function of the duration of the echo starting $3 \mathrm{~ms}$ after the onset of the emitted orientation sound $\mathbf{B}$

When the frequency shifts were kept constant at $3 \mathrm{kHz}$, that is not sinusoidally modulated, the thresholds for compensation were measured for changing echo duration and changing delay of the echoes. Two kinds of thresholds were obtained: a) the threshold of cessation of compensation when the delay was increased gradually starting from $3 \mathrm{~ms}$ or when the echo duration was shortened beginning at full overlap going to small echo durations and $b$ ) the threshold for resuming compensation when the delay was decreased or the echo duration increased. In the delay experiments both thresholds were almost equal and showed only a slight difference of about $2.5-5 \mathrm{~ms}$. The difference between the thresholds, when the echo duration was altered, was much more pronounced and also different for the individual bats. Table 1 compiles the threshold values for both measurements.

Table 1. Thresholds for the cessation of compensation, when the echo delay is increased or the echo duration decreased and thresholds for the resuming of compensation, when the echo delay is decreased or the echo duration is increased. Medium duration of orientation sounds was $50 \mathrm{~ms}$ Thresholds for cessation and resuming of compensation

\begin{tabular}{llllll}
\hline Bat & \multicolumn{2}{l}{ Echo duration (ms) } & & \multicolumn{2}{l}{ Echo delay (ms) } \\
\cline { 2 - 3 } \cline { 6 - 6 } & cessation & resuming & & cessation & resuming \\
\hline RF 7 & $<5-10$ & $>30$ & & $>22.5$ & $<20-22.5$ \\
RF 4 & $<7.5-10$ & $>30$ & & $>25$ & $<20-25$ \\
RF 8 & $<10-12.5$ & $>22.5-25$ & & $>22.5$ & $<22.5-25$ \\
\hline
\end{tabular}



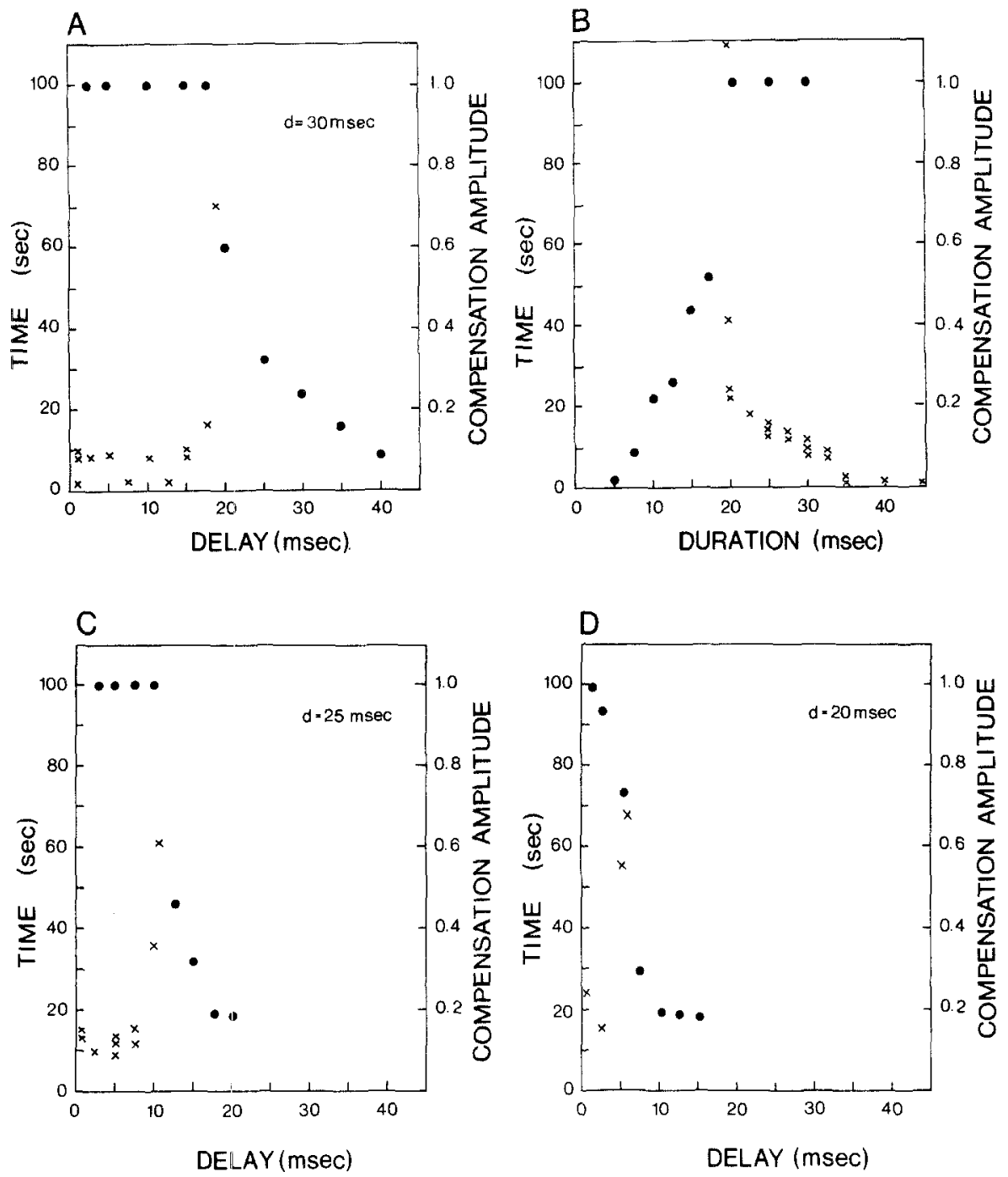

Fig. 4A-D. Playback of partially Doppler-shifted echoes: Time needed to reach full compensation (crosses) and the final compensation amplitude (points) after a constant frequency-shift of $+3 \mathrm{kHz}$ was switched on in the echo. $\mathbf{A}$ as a function of the delay of the Doppler-shifted portion of the echo (constant duration of $30 \mathrm{~ms}$ ); $\mathbf{B}$ as a function of the duration of the Doppler-shifted portion of the echo (constant delay of $\mathbf{3 m s}$ ); $\mathbf{C}$ and $\mathbf{D}$ as a function of the delay of the Doppler-shifted portion of the echo and for durations of $25 \mathrm{~ms}$ and $20 \mathrm{~ms}$ respectively

Partially Frequency Shifted Echoes. In this experiment the stimulated echoes were played back with $3 \mathrm{~ms}$ delay due to the acoustic travel time and with the same duration as the emitted orientation sound. Constant Doppler-shifts of $3 \mathrm{kHz}$ were either introduced after a delay or with a variable duration. The frequency of the non-shifted components was equal to the emitted frequency of the orientation sound. When the delay of the Doppler-shifted portion was 
increased, the compensation system needed increasingly longer times to reach full compensation and finally the Doppler-shifts were no longer compensated for completely. The times needed for compensation and the compensation amplitude finally reached are represented in the combined plots of Figure $4 \mathrm{~A}$ and $\mathrm{B}$ as functions of the delay or the duration of the frequency-shifted component. Decreasing compensation amplitude and rapidly increasing times needed to reach full compensation mark the thresholds of compensation for delay and frequency-shift duration. The thresholds lay between 17.5 and $22.5 \mathrm{~ms}$ delay for frequency-shift durations of $30 \mathrm{~ms}$ or longer. When the frequency-shift duration was smaller than $30 \mathrm{~ms}$ ( 25 and $20 \mathrm{~ms}$ in our examples) it interfered with the effects of delay on the compensation amplitude, which decayed at shorter delays as illustrated in Figure $4 \mathrm{C}$ and $\mathrm{D}$. The compensation thresholds for decreasing durations of the Doppler-shifts were found between 12.5 and $30 \mathrm{~ms}$ in different bats and varied more than the thresholds for the delay measurements. The thresholds for cessation of compensation with decreasing Doppler-shift duration were lower than those for resuming compensation when the Dopplershift duration was increased. The thresholds found for limited frequency-shifts in the echoes are consistent with those found in the experiments with segmented or artificial echoes.

\section{Discussion}

A detailed discussion on the possible function of echo overlap with the emitted orientation sounds in different species of bats was first given by Novick (1971). He calculated on the basis of data on the orientation pulse length during goaldirected flight from Schnitzler (1968), that in the first sequence of approach to the target, the bat lengthened its orientation sounds in such a way that the end of the echo always reached the ear at about the same time after the onset of the orientation sound (after about $80 \mathrm{~ms}$ and at distances between 6 and about $3.7 \mathrm{~m}$ from the landing place). At closer distances than about $3.7 \mathrm{~m}$ from the target the pulse length became shorter roughly in proportional to distance.

The pulse durations as a function of the distance to the landing perch as given by Schnitzler (1968) are represented in Figure 5 (crosses). Additionally the curve for the acoustic delay of the echoes (heavy line) and the required pulse length for different overlap times of the echoes with the emitted orientation sounds (dashed lines) are drawn in the figure. The experiments described here indicate that the compensation system begins to work at delays of about $25 \mathrm{~ms}$ corresponding to a distance between bat and target of about $4.2 \mathrm{~m}$. At this distance the length of the orientation pulses is, according to the data of Schnitzler (1968), long enough to give an overlap time which is sufficient for good compensation (overlaps longer than $30 \mathrm{~ms}$ ). In the behavioral experiments of Schnitzler on the Doppler-shift compensation (1968), however, the bats also compensated at distances between 6.5 and $4 \mathrm{~m}$ from the landing perch. This is probably due to the geometrical arrangement of the flight room used by Schnitzler, where walls protruded into the room at a distance of $4 \mathrm{~m}$ from the landing 


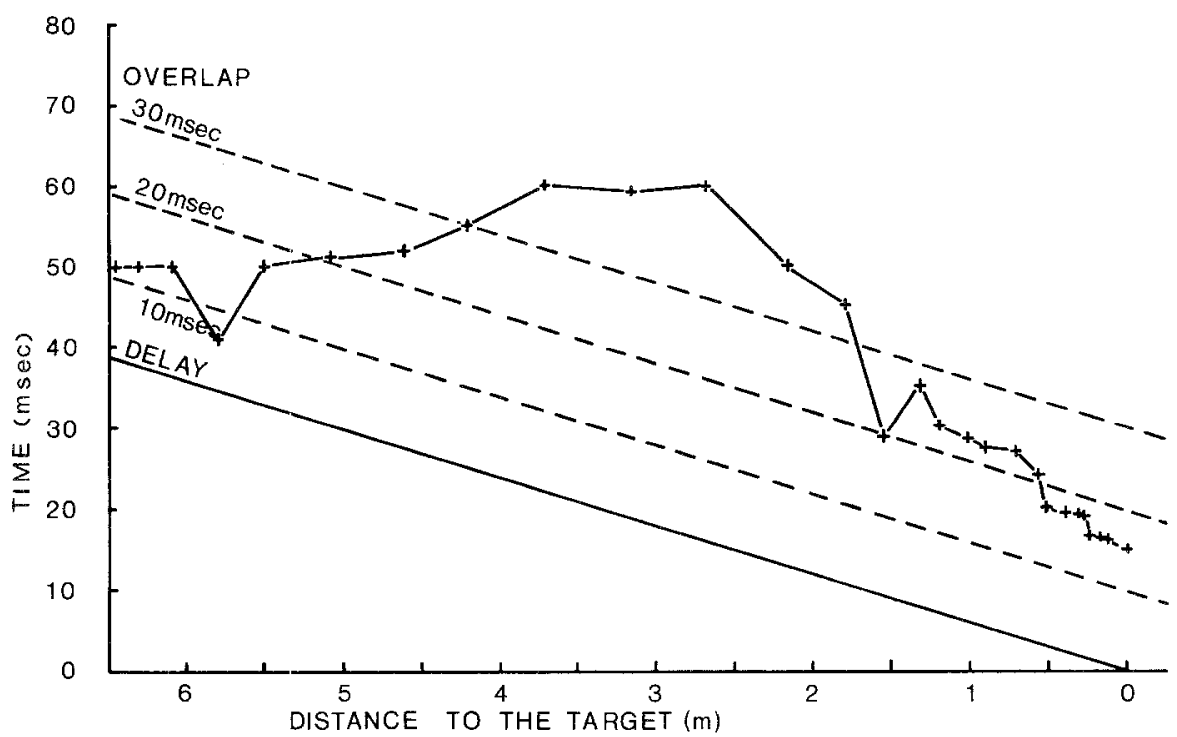

Fig. 5. Duration of orientation sounds in goal directed flight (crosses) as a function of the distance from the landing perch as measured by Schnitzler (1968). The bold line indicates the delay of the echoes due to the acoustic travel time. The dotted lines represent the durations of the sounds necessary to yield overiaps of 10,20 and $30 \mathrm{~ms}$ with the emitted orientation sound. (Figure adapted from Schnitzler (1968) by permission of the author)

place, so that the reflections from these walls could have allowed Doppler-shift compensation during the first few meters of flight.

When the bat approaches the target the delay conditions improve and the overlap time stays optimal up to about $1.7 \mathrm{~m}$ distance from the target. At that distance the echo delay is about $10 \mathrm{~ms}$. As shown in the experiments with partially frequency-shifted echoes the echolength required for good compensation of Doppler-shifts decreases when the delay of the echoes decreases. For echo lengths of 25 and $20 \mathrm{~ms}$ the maximum delays for proper compensation lie at about 10 and $5 \mathrm{~ms}$ respectively, so that compensation also works efficiently for distances below $1.7 \mathrm{~m}$ as shown in Figure 5. From this comparison of our data with those of the pulse length during target approach from Schnitzler (1968) it can be concluded that the conditions for compensation of Doppler-shifts are satisfied for distances up to $4 \mathrm{~m}$ from the target.

The intensity of the echoes relative to that of the emitted orientation sounds was kept constant in our experiments. A decrease of the echo intensity at larger distances from the target would probably reduce further the radius within which the compensation system works. The influence of the intensity parameter on the Doppler-shift compensation system in Rhinolophus ferrumequinum is an important point to be investigated.

In radar theory the inherent precision of a signal is described by the radar uncertainty function (or ambiguity function) which is a function of both the delay between transmitted signal and the returning echo and the Doppler-shift which is proportional to the radial velocity of the target. If precision is defined by the width of the uncertainty function at its half amplitude ( $-6 \mathrm{~dB}$ point) 
a single figure can be used to specify the inherent Doppler precision. In the case of a rectangular pulse with constant carrier frequency the inherent Dopplerprecision is $1.2 / \mathrm{d}$, where $\mathrm{d}$ is the duration of the pulse (Bird, 1974). An inherent Doppler precision of $1.5 \mathrm{kHz}$ would be obtained by a signal of $0.8 \mathrm{~ms}$ duration. The echo durations as the $-6 \mathrm{~dB}$ points, corresponding to a $1.5 \mathrm{kHz}$ precision in our experiment, however, ranged between 10 and $25 \mathrm{~ms}$ for different bats, i.e. at much larger echo durations. The compensation system works therefore well below the inherent Doppler precision of the signal under our experimental conditions and needs about one order of magnitude longer signals than does the optimum case.

The experiments in which durations of artificial echoes were varied gave much lower thresholds for cessation of compensation as the echo duration was shortened than for resuming compensation when the echo duration was lengthened (difference of echo length for the two thresholds was about $20 \mathrm{~ms}$ ). Updating of Doppler-shift information evidently requires shorter overlap times than for the processing of new Doppler-shift information. In this case the fact that the bat maintains the same Doppler-shift until new information is received might also be relevant (Schuller and Suga, 1976).

The accuracy of frequency measurement in Doppler-shift compensation is greatly dependent upon the duration of the echoes. As shown in the experiments with partially Doppler-shifted echoes the shortage of overlap during one sound could to some extent be compensated by a longer measuring time and repetition of the measurement in succeeding echoes. A long measuring sequence over several sounds is not, however, appropriate in normal flight as the frequency shifts can change rapidly from sound to sound and must be evaluated during one or very few sounds in the behavioral situation. As mentioned previously in the discussion the bat caters for adequate overlap by keeping its orientation sounds at an appropriate length.

The minimum duration of frequency shift necessary in the echoes is on the other hand a mechanism to protect the compensation system from interference by short transient frequency-shifts. Fast Doppler-shifts created by quick prey movements (e.g. fast changes of flight direction of the prey or wing beats of insects) would modulate the carrier frequency (CF) of the echolocation signal without interfering with the compensation for Doppler-shifts produced by the bat's flight velocity relative to a stationary background. Fast modulations of the CF component have a low hearing threshold when the CF is near the reference frequency at which the echo frequency is kept by the compensation system (Schuller, 1972).

The overlap of echo and emitted signal produces beats carrying information on the frequency difference. Suga et al. (1975) found in another CF-FM - bat (Pteronotus parnellii rubiginosus) that peripheral neurons at the level of the cochlear nucleus responded in a phase-locked manner to beats produced by delivering two simultaneous pure tones of different frequencies. Such beats were coded in the neural discharge pattern up to frequency differences of $3 \mathrm{kHz}$. As the compensation system in Rhinolophus ferrumequinum generally tracks frequency-shifts up to $4.8 \mathrm{kHz}$, the concept of decoding the Doppler-shifts by means of beats originating from the overlap between echo and orientation 
sound seems unsatisfactory. the behavioral experiments on the Doppler-shift compensation system (Schnitzler, 1973) also suggest that the bat does not primarily use the frequency difference between echo and orientation sound, but that it detects a positive frequency shift in the echo and then stabilizes the echo frequency at its reference frequency.

In a small frequency band around the reference frequency Suga et al. (1976) found neurons in the cochlear nucleus with extremely sharp tuning curves (i.e. high $\mathrm{Q}_{10}$-values) which theoretically allow a very high frequency resolution. By a set of such neurons, acting as a bank of filters, the Doppler-shift in echoes could be detected and the emission adjusted to bring the echo frequency back to the reference frequency. Such a system would not necessarily require overlap between echo and emitted orientation sound and would not limit the delay within which the frequency information from the echo could be extracted and used for the Doppler-shift compensation.

Complex interactions of excitation and inhibition are commonly found in auditory neurons on all auditory levels. The response patterns of neurons with best frequencies near the reference frequency of Rhinolophus ferrumequinum are highly dependent on the frequency and temporal relationship of two interacting sounds (Möller, 1976), so that changing delays and overlap times would influence the response pattern of such neurons.

Experiments by Legouix et al. (1973) on two-tone inhibition in the guinea pig support the hypothesis that two-tone inhibition originates from mechanical interactions of the wo tones in the cochlea. The cochlea of Rhinolophus ferrumequinum shows a considerable specialization of the basilar membrane around the reference frequency (Bruns, 1976) and the hypothesis that echo and emitted orientation sound interact mechanically in the cochlea seems attractive in this context. Critical experiments to test this hypothesis in this application remain to be done.

\section{References}

Bird, G.J.A.: Radar precision and resolution. London: Pentech Press 1974

Bruns, V.: Peripheral auditory tuning for fine frequency analysis by the CF-FM bat, Rhinolophus ferrumequinum. II. Frequency mapping in the cochlea. J. comp. Physiol. 106, 87-97 (1976)

Legouix, J.-P., Remond, M.-C., Greenbaum, H.B.: Interference and two-tone inhibition. J. acoust. Soc. Amer. 53, 409-419 (1973)

Möller, J.: Die Funktion von Hemmeinflüssen bei Neuronen der lemniscalen Hörbahn bei der Echoortung von Rhinolophus ferrumequinum. Dissertation Stuttgart 1976

Novick, A.: Echolocation in bats: some aspects of pulse design. Amer. Scientist 59, 198-209 (1971)

Pye, J.D.: A theory of echolocation by bats. J. Laryng. Otol. 74, 718-729 (1960)

Schnitzler, H.-U.: Die Ultraschall-Ortungslaute der Hufeisenfledermäuse (Chiroptera-Rhinolophidae) in verschiedenen Orientierungssituationen. Z. vergl. Physiol. 57, 376-408 (1968)

Schnitzler, H.-U.: Control of Doppler-shift compensation in the greater horseshoe bat Rhinolophus ferrumequinum. J. comp. Physiol. 82, 79-92 (1973)

Schuller, G.: Echoortung bei Rhinolophus ferrumequinum mit frequenzmodulierten Lauten. J. comp. Physiol. 77, 306-331 (1972)

Schuller, G.: The role of overlap of echo with outgoing echolocation sound in the bat Rhinolophus ferrumequinum. Naturwissenschaften 61, 171-172 (1974) 
Schuller, G., Beuter, K., Schnitzler, H.-U.: Response to frequency shifted artificial echoes in the bat Rhinolophus ferrumequinum. J comp. Physiol. 89, 275-286 (1974)

Schuller, G., Beuter, K., Rübsamen, R.: Dynamic properties of the compensation system for Doppler shifts in the bat, Rhinolophus ferrumequinum. J. comp. Physiol. 97, 113-125 (1975)

Schuller, G., Suga, N.: Storage of Doppler-shift information in the echolocation system of the "CF-FM"-bat, Rhinolophus ferrumequinum. J. comp. Physiol. 105, 9-14 (1976)

Suga, N., Simmons, J.A., Jen, P.H.-S. : Peripheral specialization for fine analysis of Doppler-shifted echoes in the auditory system of the "CF-FM" bat Pteronotus parnellii. J. exp. Biol. 63, 161-192 (1975)

Suga, N., Neuweiler, G., Möller, J.: Peripheral auditory tuning for fine frequency analysis by the CF-FM bat, Rhinolophus ferrumequinum. IV. Properties of peripheral auditory neurons. J. comp. Physiol. 106, 111-125 (1976) 